\title{
Optimization Scheme of Tradable Credits and Bus Departure Quantity for Travelers' Travel Mode Choice Guidance
}

\author{
Fang Zhou $\mathbb{D}^{1},{ }^{1}$ Jianhui Wu $\mathbb{D}^{1,2}$ Yan $\mathrm{Xu} \mathbb{D}^{1},{ }^{1}$ and Chi Yi ${ }^{1}{ }^{1}$ \\ ${ }^{1}$ School of Information Science and Technology, Hunan Institute of Science and Technology, Yueyang 414006, China \\ ${ }^{2}$ Guangxi Key Laboratory of Precision Navigation Technology and Application, Guilin University of Electronic Technology, \\ Guilin 541004, China \\ Correspondence should be addressed to Jianhui Wu; wjh_hnist@163.com
}

Received 7 October 2020; Revised 4 December 2020; Accepted 9 December 2020; Published 21 December 2020

Academic Editor: Muhammad Arif

Copyright (c) 2020 Fang Zhou et al. This is an open access article distributed under the Creative Commons Attribution License, which permits unrestricted use, distribution, and reproduction in any medium, provided the original work is properly cited.

\begin{abstract}
To analyze the influence of tradable credits and bus departure quantity on travelers' travel mode choice, this study investigated car travel and bus travel as research objects and established a two-mode day-to-day travel mode choice model based on tradable credits and bus departure quantity. To improve the guiding effect of tradable credits and bus departure quantity, an optimization scheme of tradable credits and bus departure quantity was developed with the goal of minimizing the system total travel time of car travel and the system total comprehensive cost of bus travel. Taking a test transportation network as an example, the influence of no tradable credits scheme, tradable credits scheme, and tradable credits and bus departure quantity scheme on the travelers' travel mode choice behavior was analyzed. The results showed that the tradable credits and bus departure quantity scheme could reduce the saturation of road traffic and improve bus service quality.
\end{abstract}

\section{Introduction}

How to alleviate urban traffic congestion has become a popular research topic. Currently, the methods of alleviating urban traffic congestion are mainly divided into traffic supply management and traffic demand management. In terms of traffic supply management, improving the overall supply capacity of the urban road network is the main measure to alleviate the relationship between traffic supply and demand. It mainly includes two aspects, expanding the scale and optimizing the structure of a road network, and dynamically adjusting the supply capacity of a road network based on the temporal and spatial distribution of traffic demand. The finiteness of urban land resources determines that it is unsustainable to continuously expand the scale of a road network. Therefore, optimizing its structure has become an effective way to improve the supply capacity of an urban road network. The theory and method of network design (e.g., Farahani et al. [1], Chen and $\mathrm{Xu}$ [2], and Lu et al.
[3]) provide strong support for optimizing the structure of an urban road network. In respect to dynamically adjusting network supply capacity, reversible lanes (e.g., Zhang and Gao [4], and Frejo et al. [5]) and ramp metering (e.g., Zhao et al. [6] and Kan et al. [7]) are powerful measures to optimize the network supply capacity, which can effectively alleviate the awkward situation of the coexistence of idle road resources and traffic congestion.

In terms of traffic demand management, the guidance methods of travel mode choice are important ways to alleviate traffic congestion. At present, the research on travel mode choice guidance methods is mainly concentrated on adjusting the proportion of car travel and improving the service quality of public transport. The methods for adjusting the proportion of car travel mainly include congestion pricing (e.g., Cheng et al. [8] and Zhou et al. [9]) and tradable credit schemes (e.g., Wang et al. [10] and Zang et al. [11]). Currently, the implementation of congestion pricing has a solid theoretical foundation and advanced technical 
support, including the electronic toll collection systems implemented in cities such as Singapore, London, and cities in Norway (e.g., Mark [12], Prud'homme and Bocarejo [13], and Shao et al. [14]). However, compared with congestion pricing, tradable credit schemes are a fairer and more transparent choice for travelers. Dales [15] first proposed the concept of a tradable credit scheme. Verhoef et al. [16] promoted the concept of tradable credits in traffic demand management. In recent years, the tradable credit scheme has been expanded in different ways. These mainly include heterogeneous users (e.g., Xiao et al. [17] and Wang and Huang [18]), the income effect (Wu et al. [19]), transaction costs (Nie [20]), bottleneck congestion management (Tian et al. [21]), and controlling the dynamic traffic flow evolution (e.g., Guo et al. [22] and Ye and Yang [23]).

The specific measures for improving the service quality of public transport include bus lane construction (e.g., Steiner and Irnich [24] and Zhao and Zhou [25]), urban rail transit network construction (e.g., Li et al. [26] and Wang et al. [27]), and bus route optimization (e.g., Zhang and Xu [28] and Yan et al. [29]). Although these measures can encourage travelers to choose public transport, they also increase the operating costs of public transportation. Ison [30] examined the attitudes of key stakeholder groups with respect to urban road pricing and proved that income distribution to public transport is usually preferable to investment in road infrastructure. Wu et al. [31] believed that the key to an effective travel mode guidance optimization plan is determining the appropriate fuel tax rate and the number of bus departures and then establishing a guidance optimization model based on these.

Overall, adjusting the proportion of car travel can effectively adjust the car travel demand, and improving the service quality of public transport can attract some travelers to travel by public transport, but this may increase the operating cost of public transport. To solve this problem, the suggestions are made for transport managers to levy tradable credits on car travelers and subsidize the new added operating cost of public transport through the total revenue from these tradable credits levied on car travelers. In terms of the tradable credits scheme, Miralarnaghi and Peeta $[32,33]$ proposed a multiperiod tradable credits scheme to reduce vehicular emissions. Seilabi et al. [34] supposed that the transportation authority distributed travel credits to travelers directly and instantaneously and presented an $\mathrm{AV}$-enabled tradable credits scheme to manage travel demand. Grant-Muller and Xu [35, 36] designed a tradable credits scheme based on economic measure and demonstrated that the tradable credits scheme can affect travelers' mode choice. Shirmohammadi et al. $[37,38]$ established the identity between congestion pricing and tradable credits scheme in managing network mobility and designed a tradable credits scheme to maintain the queue length of the bottleneck for homogenous travelers. Zang et al. [39] analyzed the management performance of a tradable credits scheme for high-occupancy vehicle lanes and proved that an optimal tradable credits scheme was achievable in the basic system by restricting the traffic flow of general-purpose lanes. Miralinaghi et al. [40] proposed the use of a tradable credit mechanism to manage morning commuter congestion. Tian et al. [41] demonstrated that a tradable mobility credit scheme was fairly efficient and financially sustainable in traffic demand management. These research achievements showed that the tradable credits scheme has good applicability in traffic demand management. Based on this, this study proposed an optimization scheme based on tradable credits and bus departure quantity. In this optimization scheme, the price of tradable credits was determined by the transport managers, and travelers could only purchase additional tradable credits from the transport managers. The total revenue from the scale of tradable credits in that month was used to subsidize the total operating cost of bus departure increment. The free tradable credits determined the car travel demand on the road network and affected the total revenue of transport managers selling additional tradable credits, which meant that they affected the determination of bus departure increment. Moreover, increasing bus departure quantity could improve the bus service quality and decrease the car travel demand, which meant that it affected the determination of the free tradable credits.

The paper is structured as follows. The next section establishes a two-mode day-to-day travel mode choice model based on tradable credits and bus departure quantity. Section 3 constructs an optimization model of tradable credits and bus departures quantity and proposes a solution algorithm for the optimization model. In Section 4, the feasibility of this optimization model and its solution algorithm are verified. In Section 5, the conclusions of this research are drawn.

\section{Two-Mode Day-to-Day Travel Mode Choice Model Based on Tradable Credits and Bus Departure Quantity}

To analyze the influence of tradable credits and bus departure quantity on travelers' travel mode choice, we divide travelers into car and carless travelers. In the transportation network, we assume that carless travelers can only choose to travel by bus, car travelers can choose to travel by car or bus, and car travelers who choose to travel by car must have a tradable credit on that day. Furthermore, we suppose that $q_{i j}^{o}$ is the travel demand of car travelers on OD pair $(i, j), q_{i j}^{n}$ is the travel demand of carless travelers on OD pair $(i, j), M$ is the number of days in the month, and the transport managers issue $k(0<k \leq M)$ free tradable credits to individual car travelers in that month. In other words, they issue $k q_{i j}^{o}$ is free tradable credits to group car travelers. If a car traveler needs additional tradable credits, he can only purchase tradable credits from the transport managers, and the price of tradable credit is $\rho_{t c}$. Based on this, it can be determined that the maximum number of group car travelers who purchased tradable credits is $(M-k) q_{i j}^{o}$. For readability, some of the main variables and parameters are listed in Table 1. 
TABLE 1: Main variables and parameters.

\begin{tabular}{|c|c|}
\hline Variables & Description \\
\hline$q_{i j}^{o}$ & The total demand for car travelers on OD pair $(i, j)$ \\
\hline$q_{i j}^{n}$ & The total demand for carless travelers on OD pair $(i, j)$ \\
\hline$q_{i j}^{b}(t)$ & The bus travel demand on OD pair $(i, j)$ at day $t$ \\
\hline$q_{i j}^{c}(t)$ & The car travel demand on OD pair $(i, j)$ at day $t$ \\
\hline$d_{i j}^{c}(t)$ & The total demand for car travel on OD pair $(i, j)$ from day 0 to day $t$ \\
\hline$M$ & The number of days in the month \\
\hline$k$ & Free tradable credits to individual car travelers in that month, and $0<k \leq M$ \\
\hline$y_{0}$ & The initial bus departure quantity on OD pair $(i, j)$ \\
\hline$\Delta y(t)$ & The bus departure increment on OD pair $(i, j)$ at day $t$ \\
\hline$y(t)$ & The bus departure quantity on OD pair $(i, j)$ at day $t$ \\
\hline$f_{i j}^{b}(t)$ & The bus flow on OD pair $(i, j)$ at day $t$ \\
\hline$f_{i j}^{c}(t)$ & The car flow on OD pair $(i, j)$ at day $t$ \\
\hline$f_{i j}(t)$ & The total flow on OD pair $(i, j)$ at day $t$ \\
\hline$c_{i j}^{t}(t)$ & The travel time on OD pair $(i, j)$ at day $t$ \\
\hline$c_{i j}^{c r}(t)$ & The in-bus congestion degree on $\mathrm{OD}$ pair $(i, j)$ at day $t$ \\
\hline$c_{i j}^{t c}(t)$ & The tradable credits average cost on OD pair $(i, j)$ at day $t$ \\
\hline$\pi_{i j}^{b}(t)$ & The comprehensive cost of bus travel on OD pair $(i, j)$ at day $t$ \\
\hline$\pi_{i j}^{c}(t)$ & The comprehensive cost of car travel on $\operatorname{OD}$ pair $(i, j)$ at day $t$ \\
\hline $\operatorname{ETCQ}_{i j}^{c}(t)$ & The excess tradable credits quantity on $\operatorname{OD}$ pair $(i, j)$ at day $t$ \\
\hline$z_{i j}^{b}(t)$ & The expected comprehensive cost of bus travel at day $t$ \\
\hline$z_{i j}^{c}(t)$ & The expected comprehensive cost of car travel at day $t$ \\
\hline
\end{tabular}

2.1. Comprehensive Cost of Bus Travel. Suppose that $\varsigma$ is the conversion coefficient between bus and equivalent car, and $y(t)=y_{0}+\Delta y(t)$ is the bus departure quantity on OD pair $(i, j)$ at day $t$. Then, the bus flow $f_{i j}^{b}(t)$ on OD pair $(i, j)$ at day $t$ can be expressed as

$$
f_{i j}^{b}(t)=\varsigma y(t)
$$

The total flow $f_{i j}(t)$ on OD pair $(i, j)$ at day $t$ can be written as

$$
f_{i j}(t)=f_{i j}^{c}(t)+f_{i j}^{b}(t) .
$$

Hence, the travel time $c_{i j}^{t}(t)$ on OD pair $(i, j)$ at day $t$ can be written as

$$
c_{i j}^{t}(t)=c\left(f_{i j}(t)\right),
$$

where $c(\cdot)$ is the traditional Bureau of Public Roads (BPR) link travel time function.

We define the in-bus congestion degree $c_{i j}^{c r}(t)$ as

$$
c_{i j}^{c r}(t)=\gamma\left(\frac{q_{i j}^{b}(t)}{y(t) B_{b}}\right)^{\varpi},
$$

where $\gamma$ is the conversion coefficient between the degree of bus congestion and the travel time, $B_{b}$ is the maximum passenger capacity per bus unit, and $\omega$ is the congestion coefficient.

Suppose that the waiting interval $c_{i j}^{w}(t)=1 / y(t)$, and the comprehensive cost $\pi_{i j}^{b}(t)$ of bus travel on OD pair $(i, j)$ at day $t$ is the weighted sum of travel time $c_{i j}^{t}(t)$, waiting interval $c_{i j}^{w}(t)$, in-bus congestion degree $c_{i j}^{c r}(t)$, and bus ticket price $\rho_{i j}^{s}$. It can be expressed as

$$
\begin{aligned}
\pi_{i j}^{b}(t) & =\alpha_{1} c_{i j}^{t}(t)+\alpha_{2} c_{i j}^{w}(t)+\alpha_{3} c_{i j}^{c r}(t)+\alpha_{4} \rho_{i j}^{s}, \\
& =\alpha_{1} c\left(f_{i j}(t)\right)+\alpha_{2} \frac{1}{y(t)}+\alpha_{3} \gamma\left(\frac{q_{i j}^{b}(t)}{y(t) B_{b}}\right)^{\Phi}+\alpha_{4} \rho_{i j}^{s},
\end{aligned}
$$

where $\alpha_{1}, \alpha_{2}, \alpha_{3}$, and $\alpha_{4}$ are the conversion coefficients.

2.2. Comprehensive Cost of Car Travel. It is known that group car travelers can obtain $\mathrm{kq}_{i j}^{o}$ free tradable credits in that month, and the total demand $d_{i j}^{c}(t)$ for car travel on OD pair $(i, j)$ from day 0 to day $t$ can be expressed as

$$
d_{i j}^{c}(t)=\sum_{\tau=0}^{t} f_{i j}^{c}(\tau) .
$$

When $d_{i j}^{c}(t)-\mathrm{kq}_{i j}^{o} \leq 0$, the quantity of excess tradable credits $\operatorname{ETCQ}_{i j}^{c}(t)$ on OD pair $(i, j)$ at day $t$ is equal to zero; when $0<d_{i j}^{c}(t)-\mathrm{kq}_{i j}^{o} \leq f_{i j}^{c}(t)$, the quantity of excess tradable credits $\operatorname{ETCQ}_{i j}^{c}(t)$ on OD pair $(i, j)$ at day $t$ is equal to $d_{i j}^{c}(t)-\mathrm{kq}_{i j}^{o}$; when $d_{i j}^{c}(t)-\mathrm{kq}_{i j}^{o}>f_{i j}^{c}(t)$, the quantity of excess tradable credits $\operatorname{ETCQ}_{i j}^{c}(t)$ on $\operatorname{OD}$ pair $(i, j)$ at day $t$ is equal to $f_{i j}^{c}(t)$. Thus, the relationship between $\operatorname{ETCQ}_{i j}^{c}(t)$ and $d_{i j}^{c}(t)-\mathrm{kq}_{i j}^{o}$ can be expressed as

$$
\operatorname{ETCQ}_{i j}^{c}(t)=\min \left\{f_{i j}^{c}(t), \max \left\{d_{i j}^{c}(t)-\mathrm{kq}_{i j}^{o}, 0\right\}\right\} .
$$


Considering the complexity of individual car travelers who purchase tradable credits, we propose a concept of tradable credits average cost from the perspective of group car travelers and define the tradable credits average cost $c_{i j}^{t c}(t)$ on OD pair $(i, j)$ at day $t$ as the quantity of excess tradable credits $\mathrm{ETCQ}_{i j}^{c}(t)$ multiplied by the tradable credits unit price $\rho_{t c}$ and divided by the car flow $f_{i j}^{c}(t)$. This can be expressed as

$$
c_{i j}^{t c}(t)=\frac{\rho_{t c} \operatorname{ETCQ}_{i j}^{c}(t)}{f_{i j}^{c}(t)} .
$$

Suppose that the comprehensive cost $\pi_{i j}^{c}(t)$ of car travel on OD pair $(i, j)$ at day $t$ is the weighted sum of travel time $c_{i j}^{t}(t)$, tradable credit average cost $c_{i j}^{t c}(t)$, and fuel price $\rho_{i j}$. It can be expressed as

$$
\begin{aligned}
\pi_{i j}^{c}(t) & =\beta_{1} c_{i j}^{t}(t)+\beta_{2} c_{i j}^{t c}(t)+\beta_{3} \rho_{i j}, \\
& =\beta_{1} c\left(f_{i j}(t)\right)+\beta_{2} \frac{\rho_{t c} \operatorname{ETCQ}_{i j}^{c}(t)}{f_{i j}^{c}(t)}+\beta_{3} \rho_{i j},
\end{aligned}
$$

where $\beta_{1}, \beta_{2}$, and $\beta_{3}$ are the conversion coefficients.

2.3. Two-Mode Day-to-Day Travel Mode Choice Model. Considering the influence of the travelers' travel behavior and transportation network status at day $t-1$ on the travelers' travel behavior at day $t$ (Liu et al. [42]), we suppose that carless and car travelers choose their travel mode based on their minimum expected comprehensive cost and define the expected comprehensive cost at day $t$ as the weighted sum of the expected comprehensive cost at day $t-1$ and the comprehensive cost at day $t-1$. Then, the expected comprehensive cost $z_{i j}^{c}(t)$ of car travel and the expected comprehensive cost $z_{i j}^{b}(t)$ of bus travel at day $t$ can be expressed as

$$
\begin{aligned}
& z_{i j}^{c}(t)=\phi z_{i j}^{t}(t-1)+(1-\phi) \pi_{i j}^{c}(t-1), \\
& z_{i j}^{b}(t)=\varphi z_{i j}^{b}(t-1)+(1-\varphi) \pi_{i j}^{b}(t-1),
\end{aligned}
$$

where $\phi($ or $\varphi)$ denotes a constant weight corresponding to car (or bus) travelers, which reflects the preference between the comprehensive cost of the car (or bus) travel and the expected comprehensive cost of car (or bus) travelers, and $\phi, \varphi \in[0,1]$.

Suppose that $\varepsilon_{i j}^{c}$ is the understanding error of car travel, and $\varepsilon_{i j}^{b}$ is the understanding error of bus travel. If $\varepsilon_{i j}^{c}$ and $\varepsilon_{i j}^{b}$ are independent of each other and obey the Gumbel distribution with zero means, it can be deduced that the travel mode choice behavior of car travelers satisfies the Logit model. Hence, the car travel demand $q_{i j}^{c}(t)$ on OD pair $(i, j)$ at day $t$ can be written as

$$
\begin{aligned}
q_{i j}^{c}(t) & =q_{i j}^{o} \cdot \operatorname{Pr}\left(z_{i j}^{c}(t)+\varepsilon_{i j}^{c} \leq z_{i j}^{b}(t)+\varepsilon_{i j}^{b}\right), \\
& =q_{i j}^{o} \frac{\exp \left(-\theta z_{i j}^{c}(t)\right)}{\sum_{m=\{c, b\}} \exp \left(-\theta z_{i j}^{m}(t)\right)},
\end{aligned}
$$

where $\theta(\theta>0)$ reflects the sensitivity of car travelers to the expected comprehensive cost, where a larger value of $\theta$ indicates more sensitivity and vice versa. According to Formula (12), the bus travel demand $q_{i j}^{b}(t)$ on OD pair $(i, j)$ at day $t$ can be expressed as

$$
\begin{aligned}
q_{i j}^{b}(t) & =q_{i j}^{n}+q_{i j}^{o}-q_{i j}^{c}(t), \\
& =q_{i j}^{n}+q_{i j}^{o}-q_{i j}^{o} \frac{\exp \left(-\theta z_{i j}^{c}(t)\right)}{\sum_{m=\{c, b\}} \exp \left(-\theta z_{i j}^{m}(t)\right)} .
\end{aligned}
$$

Using Formulas (10), (11), (12), and (13), the two-mode day-to-day travel mode choice model can be written as

$$
\left\{\begin{array}{c}
z_{i j}^{c}(t)=\phi z_{i j}^{t}(t-1)+(1-\phi) \pi_{i j}^{c}(t-1), \\
z_{i j}^{b}(t)=\varphi z_{i j}^{b}(t-1)+(1-\varphi) \pi_{i j}^{b}(t-1), \\
q_{i j}^{c}(t)=q_{i j}^{o} \frac{\exp \left(-\theta z_{i j}^{c}(t)\right)}{\sum_{m=\{c, b\}} \exp \left(-\theta z_{i j}^{m}(t)\right)}, \\
q_{i j}^{b}(t)=q_{i j}^{n}+q_{i j}^{o}-q_{i j}^{c}(t),
\end{array}\right\}
$$

\section{Optimization Model of Tradable Credits and Bus Departure Quantity}

3.1. Model Formulation. The guidance of travel mode choice based on tradable credits and bus departure quantity is expected to guide some car travelers to take the bus by adjusting the quantity of free tradable credits and bus departure and subsidize the total operating cost of bus departure increment with total revenue from the scale of tradable credits in that month. Based on this, an optimization model of tradable credits and bus departure quantity is proposed. It is formulated to comprehensively optimize the system total travel time of car travel and system total comprehensive cost of bus travel. It can be expressed as

$$
\min _{k, \Delta y} F=\sum_{t=1}^{M} c_{i j}^{t}(t) q_{i j}^{c}(t)+\sum_{t=1}^{M} \pi_{i j}^{b}(t) q_{i j}^{b}(t)
$$

subject to

$$
\begin{gathered}
\rho_{t c} \geq 0, y_{0} \geq 0, \Delta y \geq 0, \\
\sum_{t=1}^{M} \rho_{t c} \operatorname{ETCQ}_{i j}^{c}(t) \geq \Delta y \sigma, \\
z_{i j}^{c}(t)=\phi z_{i j}^{t}(t-1)+(1-\phi) \pi_{i j}^{c}(t-1), \\
z_{i j}^{b}(t)=\varphi z_{i j}^{b}(t-1)+(1-\varphi) \pi_{i j}^{b}(t-1), \\
f_{i j}^{c}(t)=q_{i j}^{c}(t)=q_{i j}^{o} \frac{\exp \left(-\theta z_{i j}^{c}(t)\right)}{\sum_{m=\{c, b\}} \exp \left(-\theta z_{i j}^{m}(t)\right)},
\end{gathered}
$$




$$
q_{i j}^{b}(t)=q_{i j}^{n}+q_{i j}^{o}-q_{i j}^{c}(t)
$$

where $F$ represents the objective function, and $\sigma$ is the operating cost of the unit bus departure quantity. The first item on the right side of formula (15) is the system total travel time of car travel, and the second item is the system total comprehensive cost of bus travel. Formula (16) is the nonnegative constraint on the price of tradable credit, the initial bus departure quantity, and bus departure increment. Formula (17) is the constraint to ensure that the total operating cost of bus departure increment cannot be higher than the total revenue from the scale of tradable credits in that month. Formulas (18) and (19) are the day-to-day evolution process of the expected comprehensive travel costs of car travel and bus travel, respectively. Formula (20) is the constraint of car travel demand. Formula (21) is the constraint of car travel demand.

3.2. Model Solution. Considering the complexity of the solution process for the optimization model of tradable credits and bus departure, we propose an iterative algorithm in this section. The detailed steps are described as follows:

(i) Step 1 (initialization). The days $M$ of the month, the free tradable credits $k$ of the month, the operating cost $\sigma$ of the unit departure quantity, and the initial bus departure increment $\Delta y$ are given.

(ii) Step 2: solve the two-mode day-to-day travel mode choice model, and obtain the value of $F$ and the total revenue $\sum_{t=1}^{M} \rho_{i j} \operatorname{ETCQ}_{i j}^{c}(t)$.

(iii) Step 3: calculate the maximum bus departure increment $\Delta y_{\max }=$ floor $\left(\sum_{t=1}^{M} \rho_{i j} \operatorname{ETCQ}_{i j}^{c}(t) / \sigma\right)$. If $\Delta y \leq \Delta y_{\max }$, then set $\Delta y=\Delta y+1$ and go to Step 2. Otherwise, set $\Delta y=\Delta y-1$ and go to Step 4 .

(iv) Step 4: solve the two-mode day-to-day travel mode choice model and obtain the value of $F$. If $k<M$, then set $k=k+1$ and return to Step2. Otherwise, record $F, k$, and $\Delta y$.

(v) Step 5: compare the value of $F$ under various numbers of free tradable credits and stop. Finally, $(k, \Delta y)$ is the solution of this optimization model under the minimal $F$.

Besides, the solving steps of the two-mode day-to-day travel mode choice model are described as follows:

(i) Step 1 (initialization). The total demand $q_{i j}^{n}$ of carless travelers, the total demand $q_{i j}^{o}$ of car travelers, the initial bus departure quantity $y_{0}$, the initial car flow $f_{i j}^{c}(0)$, the initial day $t$, the maximum passenger capacity $B_{b}$ per bus unit, the bus ticket price $\rho_{i j}^{s}$, the fuel price $\rho_{i j}$, the unit tradable credits price $\rho_{t c}$, and the parameters $\gamma, \emptyset, \varsigma, \alpha_{1}, \alpha_{2}, \alpha_{3}, \alpha_{4}, \beta_{1}$, $\beta_{2}, \beta_{3}, \phi$, and $\varphi$ are given. (ii) Step 2: calculate the comprehensive cost $\pi_{i j}^{b}(t)$ of the car and the travel comprehensive cost $\pi_{i j}^{c j}(t)$ of car travel according to Formulas (5) and (9), respectively.

(iii) Step 3: calculate the expected comprehensive cost $z_{i j}^{c}(t)$ of car travel and the expected comprehensive cost $z_{i j}^{b}(t)$ of bus travel according to Formulas (18) and (19), respectively.

(iv) Step 4: calculate the car travel demand $q_{i j}^{c}(t)$ and the bus travel demand $q_{i j}^{b}(t)$ according to Formulas (20) and (21), respectively.

(v) Step 5: calculate the quantity of excess tradable credits $\operatorname{ETCQ}_{i j}^{c}(t)$ according to Formula (7), and calculate the tradable credits average cost $c_{i j}^{t c}(t)$ according to Formula (8).

(vi) Step 6: if $t \geq M$, then calculate the value of $F$ and the total revenue $\sum_{t=1}^{M} \rho_{i j} \operatorname{ETCQ}_{i j}^{c}(t)$. Otherwise, set $t=$ $t+1$ and return to Step 2 .

\section{Calculations and Analysis of Numerical Example}

To validate the proposed optimization scheme of tradable credits and bus departure quantity, we suppose that there is a transportation network connecting the living area $i$ to the work area $j$, travelers have complete traffic information, and travelers can only choose to travel by car or bus in the transportation network. The transportation network will use the traditional BPR link travel time function in the following form:

$$
c_{i j}^{t}\left(f_{i j}(t)\right)=c_{i j}^{0}\left[1+0.15\left(\frac{f_{i j}(t)}{K_{i j}}\right)^{4}\right],
$$

where $c_{i j}^{0}$ is the free flow travel time on OD pair $(i, j)$, and $K_{i j}$ is the road capacity on OD pair $(i, j)$.

In this numerical example, we suppose that the total demand of car travelers $q_{i j}^{o}=4000$, the total demand of carless travelers $q_{i j}^{n}=3000$, the fuel price $\rho_{i j}=20$, the unit of tradable credits price $\rho_{t c}=200$, the initial bus departure quantity $y_{0}=130$, the conversion coefficient $\varsigma=2$, the maximum passenger capacity $B_{b}=30$, the bus ticket price $\rho_{i j}^{s}=2$, the free flow $c_{i j}^{0}=20$, and the road capacity $K_{i j}=3000$.

4.1. Calculation Results Exhibition. According to the solution algorithm of this optimization model, we select the system parameter $M=31, \alpha_{1}=1, \alpha_{2}=7200, \alpha_{3}=30$, $\alpha_{4}=1, \beta_{1}=2.2, \beta_{2}=0.5, \beta_{3}=0.5, \gamma=1, \theta=0.03, \varphi=0.5$, $\phi=0.5$, and $\sigma=32000$. The calculation results are presented as follows.

In Figure 1, we can observe that the objective function $F$ is the minimum value when the free tradable credits $k=18$. This means that the solution of the model can be derived from the proposed solution algorithm. When the free tradable credits $k=18$, the bus departure increment 


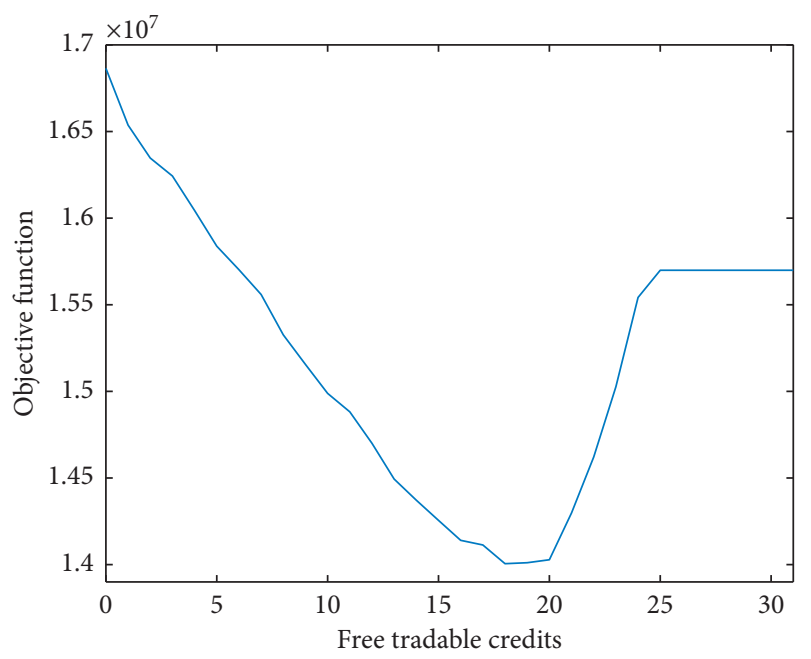

FIGURE 1: The process of objective function changing with free tradable credits.

$\Delta y=37$. It should be noted that there is no excess quantity of tradable credits and the objective function when $k \geq 24$; that is, all travelers who choose to travel by car have free tradable credits.

The system performance before and after optimization of the tradable credits and bus departure quantity are listed in Table 2. Compared to the system performance before optimization, the system total comprehensive cost $\sum_{t=1}^{M} \pi_{i j}^{b}(t) q_{i j}^{b}(t)$ of bus travel has decreased by $7.5 \%$, and the system total travel time $\sum_{t=1}^{M} c_{i j}^{t}(t) q_{i j}^{c}(t)$ of car travel has decreased by up to $26.4 \%$. This also reflects the important role of the proposed optimization scheme in traffic demand management.

4.2. Scheme Comparison and Analysis. In this section, we will compare and analyze the three schemes of no tradable credits scheme (NTCS), tradable credits scheme (TCS), and tradable credits and bus departure quantity scheme (TCBDQS). We select $k=31$ and $\Delta y=0, k=18$ and $\Delta y=0$, and $k=18$ and $\Delta y=37$ to represent the above three schemes, respectively. The results of the calculation are listed in Table 3.

In Table 3, we can observe that the road saturation under TCS and TC-BDQS is lower than that of NTCS. Compared with NTCS, road saturation under TCS has decreased by $7.7 \%$, but the saturation in buses has increased by $6.7 \%$. This shows that TCS can reduce road saturation, but it may lead to a decrease in bus service quality. Compared with TCS, road saturation under TCBDQS has decreased by $9.3 \%$, and the bus travel demand has increased by $8.9 \%$, but the saturation in buses has decreased by up to $15.1 \%$. This also reflects that TC-BDQS can not only guide some car travelers to take the bus but also improve the bus service quality.
TABLE 2: The system performance before and after optimization.

\begin{tabular}{lccc}
\hline $\begin{array}{l}\text { Optimization } \\
\text { scenario }\end{array}$ & $\sum_{t=1}^{M} c_{i j}^{t}(t) q_{i j}^{c}(t)$ & $\sum_{t=1}^{M} \pi_{i j}^{b}(t) q_{i j}^{b}(t)$ & $F$ \\
\hline $\begin{array}{l}\text { Before } \\
\text { optimization }\end{array}$ & $2.46 \times 10^{6}$ & $1.32 \times 10^{7}$ & $1.56 \times 10^{7}$ \\
After optimization & $1.81 \times 10^{6}$ & $1.22 \times 10^{7}$ & $1.40 \times 10^{7}$ \\
\hline
\end{tabular}

TABLe 3: The results of the calculation under three schemes.

\begin{tabular}{lcccc}
\hline Scheme & $\begin{array}{c}\text { Total } \\
\text { flow }\end{array}$ & $\begin{array}{c}\text { Road } \\
\text { saturation }\end{array}$ & $\begin{array}{c}\text { Bus travel } \\
\text { demand }\end{array}$ & $\begin{array}{c}\text { Saturation in } \\
\text { buses }\end{array}$ \\
\hline NTCS & 3409 & 1.136 & 3851 & 0.988 \\
TCS & 3145 & 1.048 & 4115 & 1.055 \\
TC- & 2851 & 0.950 & 4483 & 0.895 \\
BDQS & & & & \\
\hline
\end{tabular}

\section{Conclusions}

This paper established a two-mode day-to-day travel mode choice model based on tradable credits and bus departure quantity, designed an optimization scheme for these, and proposed an iterative algorithm for this scheme. Numerical examples verified the effectiveness of the proposed optimization scheme and its solution algorithm and showed the influences of NTCS, TCS, and TC-BDQS on the travelers' travel mode choice behavior. The results showed that TCS and TC-BDQS could reduce the saturation of road traffic, but TCS may lead to a decrease in bus service quality, whereas TC-BDQS could improve the bus service quality.

Beyond the above preliminary research work, there are many interesting avenues for further study. An ongoing extension of this study is to introduce the transportation condition of multiple vehicle types and multiple OD pairs in the optimization model of tradable credits and bus departure 
quantity. Another future research direction is to consider the heterogeneity of travelers' travel model choice behavior in travel demand management.

\section{Data Availability}

The data used to support the findings of this study are available from the corresponding author upon request.

\section{Conflicts of Interest}

The authors declare that they have no conflicts of interest.

\section{Acknowledgments}

This research work was supported by the National Natural Science Foundation of China (Grant nos. 61561016 and 61861008), National Key R\&D Program of China (Grant no. 2018YFB0505103), Hunan Provincial Natural Science Foundation of China (Grant no. 2019JJ50210), Scientific Research Foundation of Hunan Provincial Education Department (Grant no. 19B234), Foundation from the Guangxi Zhuang Autonomous Region (Grant nos. AC16380014, AA17202048, and AA17202033), and Guangxi Key Laboratory of Precision Navigation Technology and Application (Grant no. DH202009).

\section{References}

[1] R. Z. Farahani, E. Miandoabchi, W. Y. Szeto, and H. Rashidi, "A review of urban transportation network design problems," European Journal of Operational Research, vol. 229, no. 2, pp. 281-302, 2013.

[2] A. Chen and X. Xu, "Goal programming approach to solving network design problem with multiple objectives and demand uncertainty," Expert Systems with Applications, vol. 39, no. 4, pp. 4160-4170, 2012.

[3] H. P. Lu, X. X. Yu, and C. Z. Bian, "Model and algorithm of discrete network design problem under OD demand uncertainty," Journal of Highway and Transportation Research and Development, vol. 28, no. 5, pp. 128-132, 2011.

[4] H. Z. Zhang and Z. Y. Gao, "Optimization approach for traffic road network design problem," Chinese Journal of Management Science, vol. 15, no. 2, pp. 86-91, 2007.

[5] J. R. D. Frejo, I. Papamichail, M. Papageorgiou, and E. F. Camacho, "Macroscopic modeling and control of reversible lanes on freeways," IEEE Transactions on Intelligent Transportation Systems, vol. 17, no. 4, pp. 948-959, 2016.

[6] D. B. Zhao, X. R. Bai, F. Y. Wang, J. Xu, and W. S. Yu, "DHP method for ramp metering of freeway traffic," IEEE Transactions on Intelligent Transportation Systems, vol. 12, no. 4, pp. 990-999, 2011.

[7] Y. Kan, Y. Wang, M. Papageorgiou, and I. Papamichail, "Local ramp metering with distant downstream bottlenecks: a comparative study," Transportation Research Part C: Emerging Technologies, vol. 62, pp. 149-170, 2016.

[8] Q. Cheng, Z. Liu, and W. Y. Szeto, "A cell-based dynamic congestion pricing scheme considering travel distance and time delay," Transportmetrica B: Transport Dynamics, vol. 7, no. 1, pp. 1286-1304, 2019.

[9] B. Zhou, M. Xu, and Y. Zhang, "A trial-and-error congestion pricing method for day-to-day dynamic network flows considering travelers' heterogeneous inertia patterns," Mathematical Problems in Engineering, vol. 2019, Article ID 8514765, 17 pages, 2019.

[10] G. Wang, Z. Gao, and M. Xu, "Integrating link-based discrete credit charging scheme into discrete network design problem," European Journal of Operational Research, vol. 272, no. 1, pp. 176-187, 2019.

[11] G. Zang, M. Xu, and Z. Gao, "High-occupancy vehicle lanes and tradable credits scheme for traffic congestion management: a bilevel programming approach," PROMET-TrafficeTransportation, vol. 30, no. 1, pp. 1-10, 2018.

[12] G. Mark, "Congestion management and electronic road pricing in Singapore," Journal of Transport Geography, vol. 10, no. 1, pp. 29-38, 2002.

[13] R. Prud'homme and J. P. Bocarejo, "The London congestion charge: a tentative economic appraisal," Transport Policy, vol. 12, no. 3, pp. 279-287, 2005.

[14] J. Shao, H. Yang, X. Xing, and L. Yang, "E-commerce and traffic congestion: an economic and policy analysis," Transportation Research Part B: Methodological, vol. 83, pp. 91-103, 2016.

[15] J. H. Dales, Pollution, Property and Prices, Toronto University Press, Toronto, Canada, 1968.

[16] E. Verhoef, P. Nijkamp, and P. Rietveld, "Tradeable permits: their potential in the regulation of road transport externalities," Environment and Planning B: Planning and Design, vol. 24, no. 4, pp. 527-548, 1997.

[17] L. L. Xiao, H. J. Huang, and R. Liu, "Tradable credit scheme for rush hour travel choice with heterogeneous commuters," Advances in Mechanical Engineering, vol. 7, no. 10, pp. 1-12, 2015.

[18] J. Wang and H. Huang, "Tradable permits for traffic management with user heterogeneity," Systems Engineering-Theory and Practice, vol. 37, no. 5, pp. 1331-1338, 2017.

[19] D. Wu, Y. Yin, S. Lawphongpanich, and H. Yang, "Design of more equitable congestion pricing and tradable credit schemes for multimodal transportation networks," Transportation Research Part B: Methodological, vol. 46, no. 9, pp. 1273-1287, 2012.

[20] Y. Nie, "Transaction costs and tradable mobility credits," Transportation Research Part B: Methodological, vol. 46, no. 1, pp. 189-203, 2012.

[21] L.-J. Tian, H. Yang, and H.-J. Huang, “Tradable credit schemes for managing bottleneck congestion and modal split with heterogeneous users," Transportation Research Part E: Logistics and Transportation Review, vol. 54, pp. 1-13, 2013.

[22] R.-Y. Guo, H.-J. Huang, and H. Yang, "Tradable credit scheme for control of evolutionary traffic flows to system optimum: model and its convergence," Networks and Spatial Economics, vol. 19, no. 3, pp. 833-868, 2019.

[23] H. Ye and H. Yang, "Continuous price and flow dynamics of tradable mobility credits," Transportation Research Part B: Methodological, vol. 57, pp. 436-450, 2013.

[24] K. Steiner and S. Irnich, "Schedule-based integrated intercity bus line planning via branch-and-cut," Transportation Science, vol. 52, no. 4, pp. 882-897, 2018.

[25] J. Zhao and X. Zhou, "Improving the operational efficiency of buses with dynamic use of exclusive bus lane at isolated intersections," IEEE Transactions on Intelligent Transportation Systems, vol. 20, no. 2, pp. 642-653, 2018.

[26] M. Li, H. Wang, and H. Wang, "Resilience assessment and optimization for urban rail transit networks: a case study of Beijing subway network," IEEE Access, vol. 7, pp. 7122171234, 2019. 
[27] Y. Wang, Y. Deng, F. Ren et al., "Analysing the spatial configuration of urban bus networks based on the geospatial network analysis method," Cities, vol. 96, pp. 1-16, 2020.

[28] W. Zhang and W. Xu, "Simulation-based robust optimization for the schedule of single-direction bus transit route: the design of experiment," Transportation Research Part E: Logistics and Transportation Review, vol. 106, pp. 203-230, 2017.

[29] Y. Yan, Q. Meng, S. Wang, and X. Guo, "Robust optimization model of schedule design for a fixed bus route," Transportation Research Part C: Emerging Technologies, vol. 25, pp. 113-121, 2012.

[30] S. Ison, "Local authority and academic attitudes to urban road pricing: a UK perspective," Transport Policy, vol. 7, no. 4, pp. 269-277, 2000.

[31] J. H. Wu, Y. F. Ji, X. Y. Sun, and Y. Xu, "Guidance optimization of travelers' travel mode choice based on fuel tax rate and bus departure quantity in two-mode transportation system," Journal of Advanced Transportation, vol. 2020, Article ID 4190632, 10 pages, 2020.

[32] M. Miralinaghi and S. Peeta, "Promoting zero-emissions vehicles using robust multi-period tradable credit scheme," Transportation Research Part D: Transport and Environment, vol. 75, pp. 265-285, 2019.

[33] M. Miralinaghi and S. Peeta, "Design of a multiperiod tradable credit scheme under vehicular emissions caps and traveler heterogeneity in future credit price perception," Journal of Infrastructure Systems, vol. 26, no. 3, pp. 1-13, 2020.

[34] S. E. Seilabi, M. T. Tabesh, A. Davatgari, M. Miralinaghi, and S. Labi, "Promoting autonomous vehicles using travel demand and lane management strategies," Frontiers in Built Environment, vol. 6, pp. 1-14, Article ID 560116, 2020.

[35] S. Grant-Muller and M. Xu, "The role of tradable credit schemes in road traffic congestion management," Transport Reviews, vol. 34, no. 2, pp. 128-149, 2014.

[36] M. Xu and S. Grant-Muller, "Trip mode and travel pattern impacts of a tradable credits scheme: a case study of Beijing," Transport Policy, vol. 47, pp. 72-83, 2016.

[37] N. Shirmohammadi, M. Zangui, Y. Yin, and Y. Nie, "Analysis and design of tradable credit schemes under uncertainty," Transportation Research Record: Journal of the Transportation Research Board, vol. 2333, no. 1, pp. 27-36, 2013.

[38] N. Shirmohammadi and Y. Yin, "Tradable credit scheme to control bottleneck queue length," Transportation Research Record: Journal of the Transportation Research Board, vol. 2561, no. 1, pp. 53-63, 2016.

[39] G. Z. Zang, M. Xu, and Z. Y. Gao, "High-occupancy vehicle lane management with tradable credit scheme: an equilibrium analysis," Transportation Research Part E: Logistics and Transportation Review, vol. 144, pp. 1-36, 2020.

[40] M. Miralinaghi, S. Peeta, X. He, and S. V. Ukkusuri, "Managing morning commute congestion with a tradable credit scheme under commuter heterogeneity and market loss aversion behavior," Transportmetrica B: Transport Dynamics, vol. 7, no. 1, pp. 1780-1808, 2019.

[41] Y. Tian, Y.-C. Chiu, and J. Sun, "Understanding behavioral effects of tradable mobility credit scheme: an experimental economics approach," Transport Policy, vol. 81, pp. 1-11, 2019.

[42] S. X. Liu, W. S. Chen, Q. Y. Chi, and H. Yan, "Day-to-day dynamical evolution of network traffic flow with elastic demand," Acta Physica Sinica, vol. 66, no. 6, pp. 1-15, Article ID 060501, 2017. 